\title{
Hemangioendothelioma of the Spinal Cord with Intramedullary Extension
}

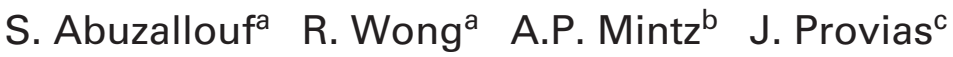 \\ a Department of Radiation Oncology, Hamilton Regional Cancer Centre, and ${ }^{\mathrm{b}}$ Departments of Neurosurgery, \\ Pathology and Molecular Medicine, Neuropathology, Hamilton General Hospital, McMaster University, \\ Hamilton, Canada
}

\author{
Key Words \\ Hemangioendothelioma · Spinal cord • \\ External beam radiotherapy
}

\begin{abstract}
Objective: Hemangioendotheliomas (HE) are vascular neoplasms that rarely involve the neuraxis. We report a rare case in the literature of intradural HE of the spinal cord with intramedullary extension. Clinical Presentation and Interventions: A 41-year-old gentleman presented with low back pain, numbness and urinary retention. Imaging revealed a spinal tumor causing complete blockage at the level of $\mathrm{T}_{12}$. The tumor was resected and postoperative radiotherapy was delivered for residual disease. No disease was seen on MRI after 48 months of clinical and radiological follow-up. Conclusion: Complete excision of HE is the treatment of choice. Radiotherapy may play a role in the management of this lesion.
\end{abstract}

Copyright (C) 2005 S. Karger AG, Basel

\section{Introduction}

Hemangioendothelioma (HE) is a vascular neoplasm composed of endothelial cells and is considered to be of an intermediary pathology between hemangioma and frankly malignant angiosarcoma [1]. These tumors can occur in almost all locations but rarely involve the neuraxis. Three histologic subgroups including epithelioid, spindle cell and malignant endovascular HE have been defined [2]. The etiology is unknown, but it may be associated with vascular malformations involving other organs. It can also be associated with hemolytic anemia, thrombocytopenia and coagulopathy [3].

We describe a rare case in the literature of an intraspinal (intradural with intramedullary extension) HE occurring in a young man with a complete resolution of residual tumor seen on MRI after postoperative radiotherapy.

\section{Case Report}

In March of 1999, a 41-year-old gentleman presented with a 3 months' history of low back pain that radiated to his right leg. His pain developed acutely and was predominantly right-sided. The pain progressed despite analgesics and physiotherapy. One month later, he developed numbness in the right inner thigh, scrotum and perianal region. He also developed urinary retention with dribbling, difficulty with defecation and abdominal distention.

Clinical examination revealed a decreased sensation to light touch, pinprick and temperature in the right gluteal and perianal region. Deep tendon reflexes in the knee and ankle were absent. An urgent CT-myelogram showed a complete blockage at the level of $\mathrm{T}_{12}-\mathrm{L}_{1}$. An irregular, lobular, markedly contrast enhancing mass opposite to $T_{12}$ vertebral body was seen on MRI (fig. 1).

\section{KARGER}

Fax +41613061234 E-Mail karger@karger.ch www.karger.com
Dr. Raimond Wong

Radiation Oncology Department, Hamilton Regional Cancer Centre 699 Concession Street

Hamilton, Ontario (Canada)

Tel. +19053879495, Fax +1905 575 6323, E-Mail raimond.wong@hrcc.on.ca 

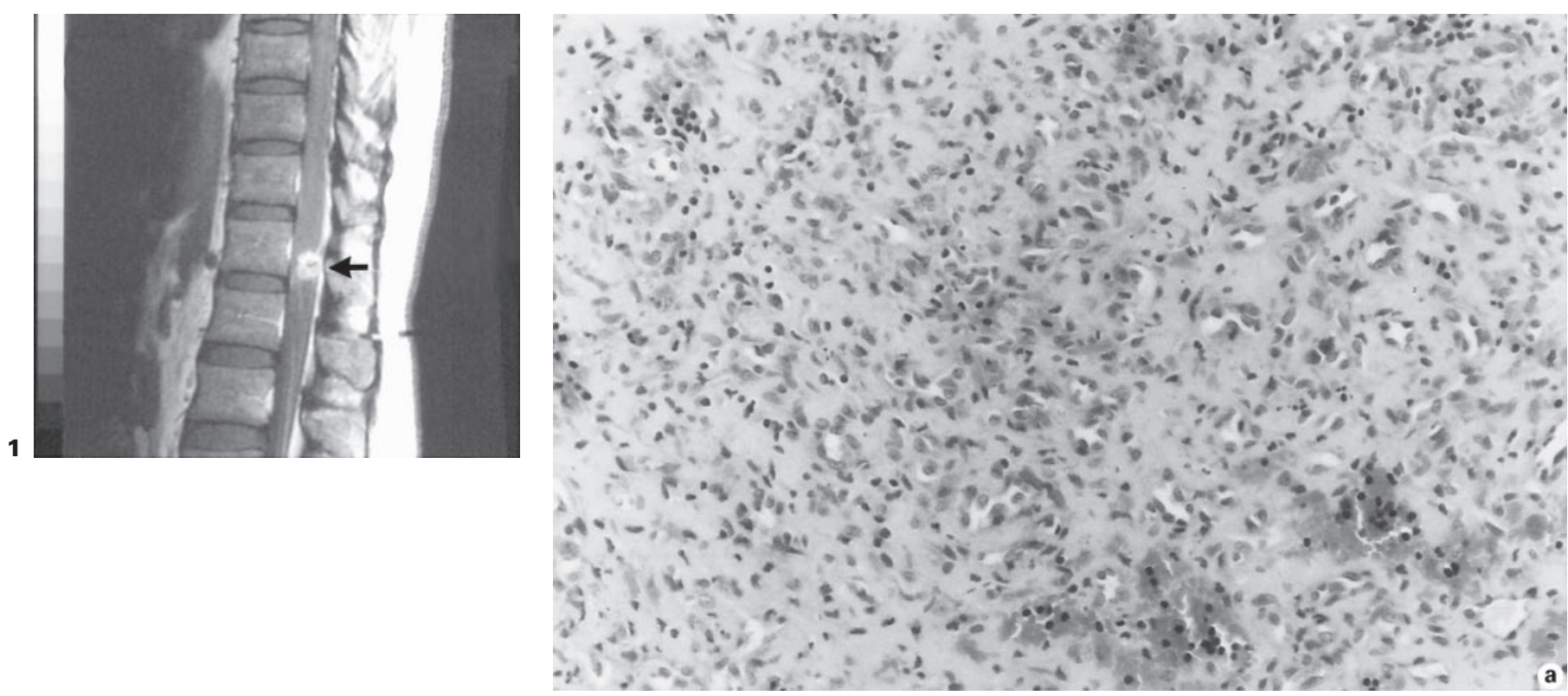

Fig. 1. Preoperative sagittal $T_{1}$-weighted postcontrast MRI scans showing an irregular, lobular, markedly enhancing mass (arrowed) opposite the $\mathrm{T}_{12}$ vertebral body.

Fig. 2. a Histology of the lesion showing an irregular anastomosing network of vascular channels with plump lining endothelial cells and a similar intervening cell population in more solid regions. HE. Original magnification $\times 100$. b Immunohistochemistry of the lesion, showing positivity for endothelial cell marker CD34, outlining the vascular network and many of the intervening cells. Immunohistochemistry, antibody to CD34. Original magnification $\times 250$.

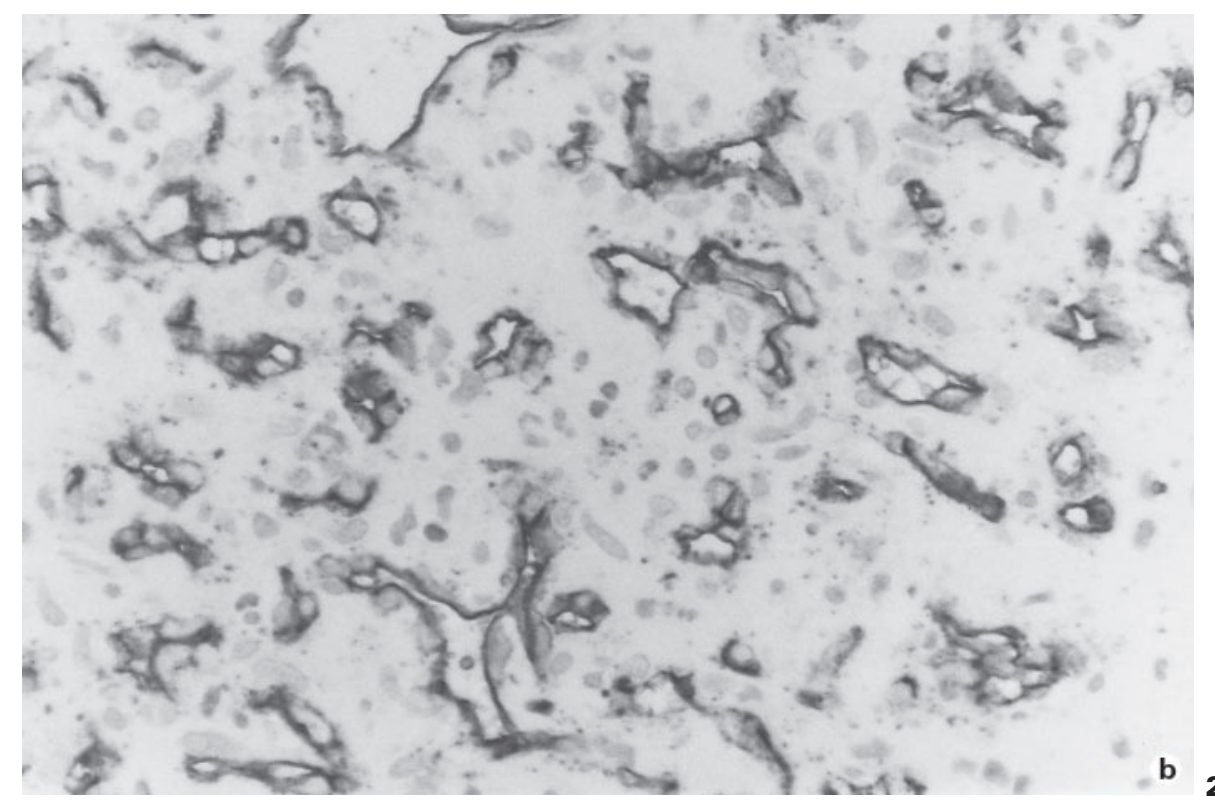

The patient then underwent a laminectomy from $\mathrm{T}_{12}-\mathrm{L}_{1}$. After removal of the overlying bone, the dura on the right-hand side was found to have a slightly bluish discoloration. The dura was tense with a firm mass palpable beneath the surface. Upon opening of the dura, a blue-black tumor was identified with many vessels present on its surface. The tumor was attached to the filum terminale. A biopsy was taken and the tumor was found to be quite vascular and firm. After severing the filum and dissecting the surrounding roots from the tumor, dissection proceeded superiorly, separating the tumor from the pial surface of the spinal cord. Debulking of the tumor was accomplished by using an ultrasonic aspirator. Near the superior portion, the tumor was found to extend into the spinal cord. This intramedullary portion was care- fully dissected from the spinal cord. The tumor was separable from the spinal cord in most places but there was no well-developed capsule. Using bipolar coagulation and ultrasonic aspirator, gross removal of all visible tumor was completed. Augmentation duroplasty was then performed with a dura graft and the wound was closed in layers.

The pathology specimen consisted of multiple fragments of soft hemorrhagic tissue measuring in aggregate approximately $2.0 \times$ $3.0 \mathrm{~cm}$. This was fixed in $10 \%$ buffered formalin. Histologic sections were stained with hematoxylin and eosin (HE) in the standard fashion, as well as with an extensive immunohistochemical panel. Immunohistochemical stains included endothelial cell markers, factor VIII, CD34, CD31, vimentin, glial fibrillary acidic protein, 


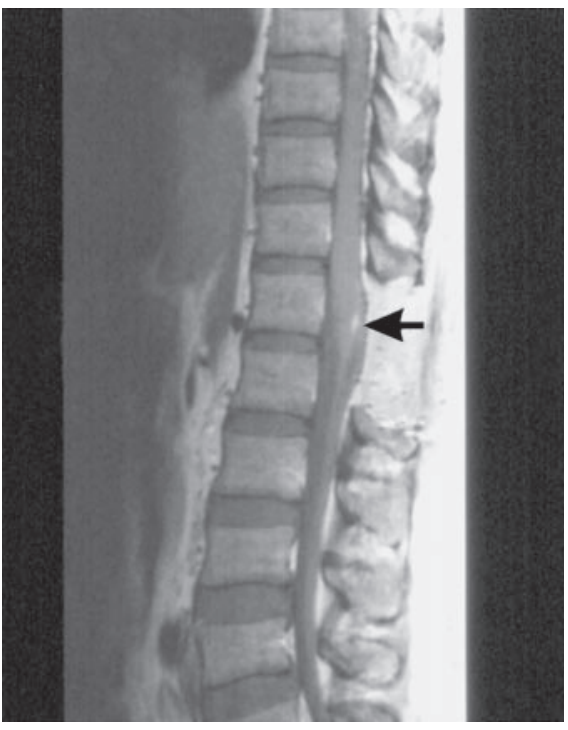

Fig. 3. Postoperative sagittal $\mathrm{T}_{1}$-weighted postcontrast MRI scans showing residual enhancing tissue (arrowed) at the $\mathrm{T}_{12}$ level.

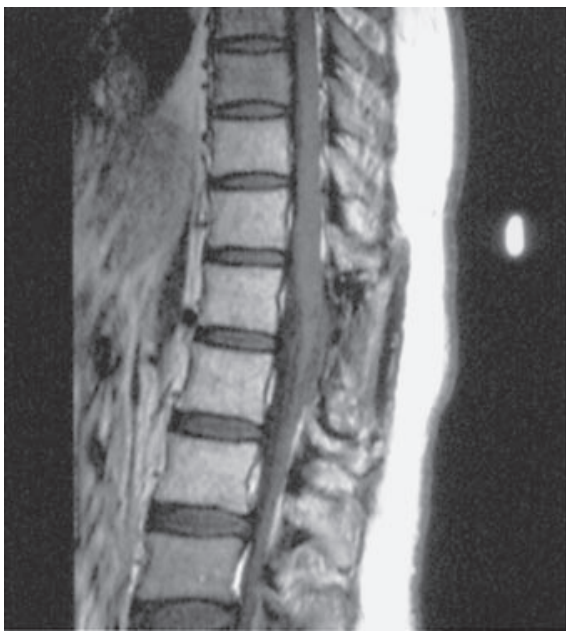

Fig. 4. Postradiotherapy sagittal $T_{1}$-weighted MRI scans showing complete resolution of the residual enhancing tissue seen at the $\mathrm{T}_{12}$ level (48 months after treatment).

keratins CAM5.2 AE1/AE3, neuron-specific enolase (NSE), S100, epithelial membrane antigen, and synaptophysin.

The lesion consisted of a vascular tumor composed largely of an irregular aggregate of vascular spaces with an endothelial cell lining and an intervening population of similar endothelial cells (fig. 2a). Vascular channels varied in caliber from very small capillary type vessels to larger more ectatic back-to-back vascular arrangements.
Varying degrees of intervening collagenous stromal fibrous tissue and focal area of myxoid stromal change were present. There was evidence of previous intratumoral hemorrhage with hemosiderin deposits. The endothelial cells ranged from bland to focally more plump, large and atypical forms that were polygonal to spindled. In areas, individual cells showed intracellular cytoplasmic vacuoles. Mitotic activity was not present. The immunohistochemical profile confirmed the endothelial and vascular nature of the lesion with both the intervening as well as vascular space lining cells showing positivity for the endothelial cell markers factor VIII, CD34, CD31 and vimentin (fig. 2b). The cells were negative for keratin, NSE, S100, EMA, GFAP and synaptophysin. A diagnosis of hemangioendothelioma was made.

Postoperatively, the patient's urinary and bowel symptoms resolved but he continued to have mild perianal numbness and left hip flexor weakness for which he used a cane. A postoperative MRI demonstrated a small enhancing nodular area at the right posterior conus at the $\mathrm{T}_{12}$ level, measuring less than $1 \mathrm{~cm}$ in length (fig. 3). This was adjudged to represent residual disease on radiology review and radiotherapy was offered to improve local control. He received a total of $50.4 \mathrm{~Gy}$ in 28 fractions over 5.5 weeks $(1.8 \mathrm{~Gy}$ per fraction). He was followed on a regular basis with clinical examination and MRI scans. The patient is doing well with no radiation toxicity and no residual disease on the MRI at 48 months' follow-up (fig. 4).

\section{Discussion}

HE is a neoplasm composed of endothelial cells, and is considered to represent a neoplasm of intermediate or borderline malignant potential [1]. More recently, histologic subtypes, including epithelioid, spindle cell and polymorphous forms, have been described [2]. The present case conforms best to the polymorphous subtype of HE demonstrating some variability in morphologic pattern with spindled cell areas, more polygonal epithelioid areas, as well as variable vascular density within the lesion.

The treatment of choice for spinal HE is complete excision of the tumor. Although radiation has been used as an adjuvant treatment, the role of irradiation has not been clearly defined. In 1980, Pearl et al. [4] treated a benign epidural $\mathrm{HE}$ at $\mathrm{T}_{8}$ spine with surgery and postoperative radiotherapy (radiation $=30 \mathrm{~Gy} / 10$ fraction) with good results. Mahdavi et al. [5] reported an intradural-extramedullary lesion that recurred twice and was irradiated after the third resection. The first local recurrence occurred 8 months after the first operation. The second local recurrence happened at about 4 months from the second surgery. However, when radiotherapy was delivered postoperatively after the third resection, no disease was seen on MRI for 28 months. Unfortunately, it is unclear whether partial or gross total resection was performed. Ronca- 
roli et al. [6] recently reported a case of primary HE from the spinal cord that did not recur 14 months after a complete resection. Preoperative radiotherapy has been used to remove a huge tumor completely [7].

In the pediatric population, there is an extensive experience with the use of interferon-alpha. It has been used in treating HE with good results [3]. Other chemotherapeutic agents have been used including ifosfamide, etoposide, platinum and cyclophosphamide with varying responses [8-10].

The case presented in this report is unique in many aspects. Firstly, besides being a rare disease involving a rare site (spinal cord), the lesion was intradural with intramedullary extension. Previous case reports involve tumor in the intradural and extramedullary sites [5]. Apart from two reports $[6,11]$, a literature review has failed to identify reports on primary spinal HE with intramedullary or partially intramedullary involvement as in the current case report. Secondly, postoperative radiotherapy was offered to the patient rather than observation. This decision was based on the documentation of residual disease and the higher risk of morbidity with repeated surgery in this patient. Although the role of radiotherapy in the management of spinal HE is still unclear, it would ap- pear to offer some advantage over surgery alone as it would seem to diminish the chances of recurrences in the operated cases [5, 11]. A recent report by Roncaroli et al. [6] suggested observation alone after surgery without adjuvant therapy, however, the follow-up period of 14 months in the reported case was comparatively short. The present case is similar to that reported by Abdullah et al. [11] where postoperative limited field radiation was given although there was no interferon therapy offered to our patient after radiation. Similar to that report, our patient has no evidence of progression of disease at 48 months' follow-up. At this point, no radiation toxicity has been detected in our patient. In fact, there was no evidence of recurrent disease as reported in the most recent MRI.

\section{Conclusion}

The optimum treatment for HE has not been well established, maximal surgical resection probably remains the main modality of therapy. However, postoperative radiotherapy may play a role in the management of intraspinal HE especially in partially resected or recurrent cases.

\section{References}

1 Brennan JW, Midha R, Ang LC, Perez-Ordonez B: Epithelioid hemangioendothelioma of the spine presenting as cervical myelopathy: case report. Neurosurgery 2001;48:11661169.

-2 Weiss SW, Enzinger FM: Epithelioid hemangioendothelioma: a vascular tumor often mistaken for a carcinoma. Cancer 1982;50:970981

-3 Deb G, Donfrancesco A, Ilari I, De Sio L, Milano GM, Ghitti C, Fontana G, Sandri A, Helson L: Hemangioendothelioma: successful therapy with interferon-alpha: a study in association with the Italian Pediatric Hematology/ Oncology Society (AIEOP). Med Pediatr Oncol 2002;38:118-119.

-4 Pearl GS, Takei Y, Tindall GT, O’Brien MS, Payne MS, Hoffman JC: Benign hemangioendothelioma involving the central nervous system: 'strawberry nevus' of the neuraxis. Neurosurgery 1980;7:249-256.
Mahdavi Z, Grafe MR, Ostrup R, Kormanik $\mathrm{P}$, Chamberlain MC: Spindle cell hemangioendothelioma of the spinal cord. J Neurooncol 1996;27:231-234.

-6 Roncaroli F, Scheithauer BW, Papazoglou S: Primary polymorphous hemangioendothelioma of the spinal cord: Case report. J Neurosurg 2001;95(suppl 1):93-95.

$>7$ Kubota T, Sato K, Takeuchi H, Handa Y: Successful removal after radiotherapy and vascular embolization in a huge tentorial epithelioid hemangioendothelioma: a case report. J Neurooncol 2004;68:177-183.

\footnotetext{
8 Sharif K, English M, Ramani P, Alberti D, Otte JB, McKiernan P, Gosseye S, Jenny M, de Ville de Goyet J: Management of hepatic hemangioendothelioma in children: what option? $\mathrm{Br} \mathrm{J}$ Cancer 2004;90:1498-1501.

-9 Pinet C, Magnan A, Garbe L, Payan MJ, Vervloet D: Aggressive form of pleural epithelioid hemangioendothelioma: complete response after chemotherapy. Eur Respir J 1999;14:237238.

10 Lezama-del Valle P, Gerald WL, Tsai T, Meyers P, La Quaglia MP: Malignant vascular tumors in young patients. Cancer 1998;83:16341639.

11 Abdullah JM, Mutum SS, Nasuha NA, Biswal BM, Ariff AR: Intramedullary spindle cell hemangioendothelioma of the thoracic spinal cord - case report. Neurol Med Chir (Tokyo) 2002;42:259-263.
} 